\title{
Vesical hydatic cyst: an unusual localization of the hydatidosis of the child
}

\begin{abstract}
Hydatidosis is endemic in our country. Vesical hydatic cyst is rare. We report a case of isolated hydatid localization at the bladder. She was a 5 years old girl, admitted for prerectal isolated mass. The etiological diagnosis had objectified a cystic mass pushing back the bladder dome, evoking a cyst of the urachus or the ovary. Surgical exploration had found a cystic mass adhering to the bladder dome. The mass and its implantation base were excised. The surgery was simple. Histopathological examination concluded that the vesical dome had a hydatid cyst. The hydatid cyst is rare. The clinical expression of this localization is variable. It can pose diagnostic and therapeutic problems. You have to know how to think about it before any cystic mass of the pelvis. Primary prevention remains the preferred route to control this endemic.
\end{abstract}

Keywords: endemic, child, hydatid cyst, vesical, prevention
Volume 8 Issue 4 - 2018

\author{
EE Kamili, ${ }^{1,2}$ F Bennaoui, ${ }^{2}$ K Fouraiji,, ${ }^{1,2}$ M \\ Oulad Saiad ${ }^{1,2}$ \\ 'Department of Pediatric Surgery, Mother-Child Hospital, \\ Mohamed VI Teaching Hospital, Morocco \\ ${ }^{2}$ Medicine School, Cadi Ayyad University, Morocco
}

Correspondence: Fatiha Bennaoui, Medicine School, Cad Ayyad University, Morocco, Email fatihabennaoui@yahoo.fr

Received: July 28, 2018 | Published: August 20, 2018

\section{Introduction}

Hydatidosis is endemic in our country, where it is a public health problem. The involvement of the bladder is in relation to the pulmonary and hepatic localizations. Primary pelvic involvement is by blood. Ultrasound and CT scan are necessary in diagnosis, but this diagnosis is sometimes made only intraoperatively. The treatment is surgical. Post-operative surveillance is based on immunology and medical imaging.

We report a case of isolated hydatid localization, at the bladder, hospitalized in surgical department, University Hospital, Mohamed VI, Marrakesh. The objective of our study is to identify the diagnostic and therapeutic difficulties related to this localization.

\section{Case report}

A five years old girl, with no particular pathological history. She was hospitalized for an abdominopelvic mass, which had been going on for three months. Clinical examination revealed a medial and oval hypogastric mass, with regular contours, measuring 10 $\mathrm{cm}$ by $6 \mathrm{~cm}$, fixed in relation to the deep parietal site. The rest of the clinical examination was normal, including no hepatomegaly or pleuropulmonary signs. The diagnosis was that of a tumor of the urogenital sinus.

The abdominal ultrasound requested in first intention had mounted a cystic mass, pushing laterally the bladder and could correspond either to a diverticulum of the bladder, or to a cyst of the ovary (Figure 1). CT scan was also performed and had objectified a cystic mass under the umbilical, median compressing behind the bladder dome with overflow to the right of the bladder horn, evoking a cyst of the urachus or an ovarian cyst (Figure 2). Surgical exploration revealed a cystic mass forming part of the bladder dome, evoking a cystic tumor of the urachus. The mass was removed by removing the implantation base from the bladder dome. The postoperative consequences were simple. The pathological report concluded that there was a hydatid cyst of the bladder dome.

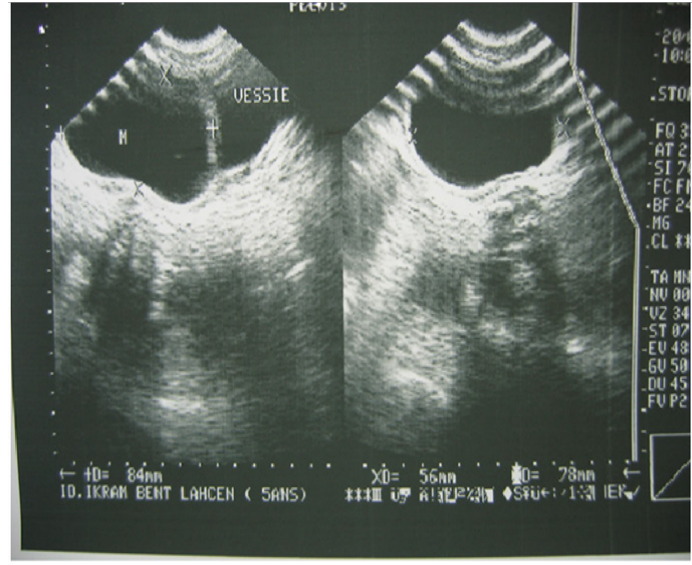

Figure I Ultrasound image showing a cystic mass pushing back the bladder laterally.

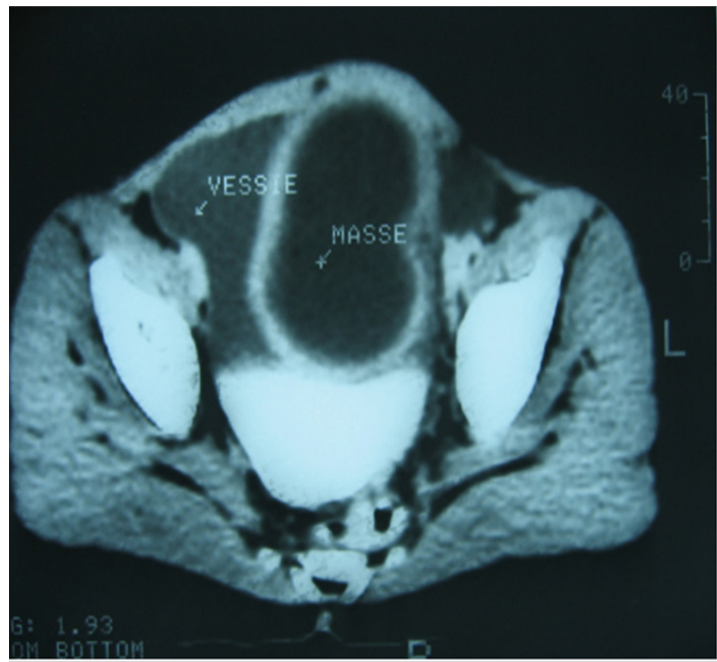

Figure 2 CT scan: an umbilical cystic mass, median compressing back the bladder dome. 


\section{Discussion}

Hydatidosis is common in Morocco; where it is endemic. It can be localized in any localization of the body, as soon as the hepatic and pulmonary filters are exceeded. ${ }^{1}$

The involvement of the urogenital tract remains among the rare and misleading clinical and anatomical aspects, especially in the absence of a context or a history of hydatidosis. It is still exceptional in the bladder. $^{2}$ A dual etiopathogenic mechanism could explain this localization. First; primary haematogenous dissemination of hexacanth embryos, ${ }^{3}$ and secondly, secondary involvement from a pelvic localization after fissuring or rupture of a hydatid cyst. ${ }^{4}$ The first hypothesis remains the most probable in our case because of the absence of other hydatid localizations. Exceptionally, two other routes can be taken by the larvae: the lymphatic route, which directly reaches the thoracic duct through the intestinal chyliferae, or the venous pathway, following Retzius venous systems and Schmiedel anastomoses.

Bladder localization is characterized by its slow evolution and clinical latency for a long time. ${ }^{6}$ Clinical manifestations appear only when the cyst reaches a considerable volume, becoming palpable and realizing a compression of the neighboring organs, responsible for the signs of vesical and rectal imprints. ${ }^{1}$ Low back pain associated with obstructive ureteric compression is rare. ${ }^{7}$ Urinary signs can range from simple dysuria, to acute urine retention, to even kidney failure.

Hydatidosis is a pathognomonic but exceptional sign, reflecting the opening of the cyst in the bladder. ${ }^{9}$ The physical examination discovers a hypogastric mass that remains unchanged after bladder emptying. The digital rectal perceives the inferior pole renit of mass. ${ }^{10}$ The diagnosis of hydatid cyst of the bladder can be easily evoked in front of the signs of orientation: On the clinical level, the interrogation researches the notion of contact with the dogs, the notion of previous intervention on a hepatic hydatidosis, the existence of an acute accident evoking rupture or fissuring and hydaturia. ${ }^{9}$ In their absence, this diagnosis can be difficult at the clinical stage.

Our patient did not have the notion of contact with dogs, she has no history of hydatid disease. There is no specific clinical sign of pelvic hydatid cyst.

Abdominal radiography without preparation may show calcifications that are suggestive but not specific. The diagnosis is based primarily on the abdominal-pelvic ultrasound that locates the mass, to define its character, to look for other locations and abdominal peritoneal retro and appreciate the repercussion on the upper urinary tract. ${ }^{11}$ Moreover, it makes it possible to define 5 types of hydatid cyst according to the classification of Gharbi and al. ${ }^{12}$ Ultrasound images of type II, III and V cysts generally do not raise doubts about their hydatid origin. The image of a vesicular or type I cyst can be confusing with an ovarian or uracic cyst. Type IV gives images that are difficult to distinguish from an ovarian tumor. The limits of ultrasound are the difficulty to demonstrate all the hydatid localizations of the abdominopelvic cavity and to establish the starting point of the cyst, especially when it is bulky. ${ }^{13}$ In these cases, CT scan is indispensable; it is very sensitive for the detection of calcifications, girls' vesicles and better specifies the relations with the neighboring organs, as well as a precise enumeration of the cysts. ${ }^{6}$ In case of strong suspicion, cystoscopy allows the diagnosis. ${ }^{14} \mathrm{X}$-rays of the thorax make it possible to search for possible pulmonary localizations. ${ }^{15}$ On the biological analysis: hyper eosinophilia is not constant and hydatid serology is often negative in this localization. ${ }^{6}$

In our case, CT and chest X-ray showed no other localization, which favors a primary bladder location. The treatment of hydatid cyst of the bladder is surgical. It consists of a total or partial kystoperi-cystectomy in case of contiguity to dangerous areas. The extra peritoneal approach is preferable to avoid contamination of the abdominal cavity. ${ }^{4}$ The approach must be wide. The field of operation must be protected by fields soaked with scolicide. The total excision of the cyst remains the ideal method, which was the case in our patient. Fistulization in the bladder represents a mode of healing, instillation of hypertonic saline may be sufficient, thus changing surgery in patients at high surgical risk. ${ }^{14}$ The medical treatment based on imidazole administered pre and postoperatively, is indicated in cases of multiple cysts with incomplete excision or associated diffuse intraperitoneal localization, and to prevent secondary localizations. Therapeutic success corresponds to the disappearance or regression of or cysts, their calcification or the absence of relapse. Postoperative monitoring is an integral part of management. It is based on clinical, immunology and medical imaging.

\section{Conclusion}

Hydatic cyst of the bladder is a rare disease. The clinical manifestations are dominated by micturition disorders and the discovery of an abdominal mass. Ultrasound and serology allow the diagnosis to be made in the majority of cases. In case of doubt, CT can be very useful. The treatment is primarily surgical. It remains to emphasize the primary prophylactic measures, which alone will reduce the incidence of hydatidosis in our country and therefore its medical and socio-economic impact.

\section{Acknowledgments}

None.

\section{Conflict of interest}

The author declares that they have no conflict of interests.

\section{References}

1. Moussaoui EL, Aboutaieb R, Joual A. Retrovesical hydatid cyst in children: report of 2 cases. J Urol. 1994;100:101-104.

2. Rizza G, Bartolotta G, Vacirca F. Retrovesical hydatid cyst fistulized into the bladder, associated with multiple hepatic and epiploic hydatidosis. Chir Ital. 1980,32:1394-1414.

3. Angulo JC, Escribano J, Diego A, et al. Isolated retrovesical and extrarenal retroperitoneal hydatidosis: clinical study of 10 cases and literature review. J Urol. 1998;159(1):76-82.

4. Biyabani SR, Farhat A, Shehzad G. Unusual presentations of hydatid disease of the urinary tract. $J$ Urol. 2000;163:896-898.

5. Benchekroun A, Hachimi M, Marzouk M, et al. Retro-peritoneal hydatid cysts: 3 new cases. Acta. Urol. Belg. 1987;55(4):522-527.

6. Horchani A, Nouira Y, Kbaier I, et al. Hydatid cyst of the kidney. A report of 147 controlled cases. Eur Urol. 2000;38(4):461-467.

7. Bennani S, El Mrini M, Raji A, et al. Retro-vesical and retroperitoneal hydatid cysts isolated: about five cases. Ann Urol. 1992;26(6-7):344 349 .

8. Deodhar SD, Patel VC, Kirloskar MS. Hydatid disease of urinary bladder 
(a case report). J Postgrad Med. 1986;32(1):46-48.

9. Baba A, Chaib A, Khairi H. Epidemiological profile of pelvic hydatidosis: about 15 cases. J Gynécol Obstét Biol Reprod. 1991; 20:657-660.

10. Jamal Bouihi, Houda Moustaide, Bouchra E, et al. Primary pelvic hydatid cyst: about a case. Pan Afr Med J. 2016;25:239.

11. Cherkaoui MM, Nassar I, Jroundi L, et al. Hydatid disease of the urinary bladder: a case report. J Radiol. 2002;83:45-46.

12. Saqui AE, Aggouri M, Benzagmout M, et al. Hydatid cyst of the posterior fossa. Pan Afr Med J. 2017.

13. Hafsa C, Golli M, Kriaa S, et al. Retrovesical hydatid cyst in children: report of 3 cases. J Radiol. 2007;88:968-971.

14. Sallami S, Nouira Y, Kallel Y, et al. Intravesical hydatid cyst. Urology. 2005;66(5):1110.

15. Boufettal R, Lefryiekh MR, Fadil A, et al. Primitive pelvic hydatial cyst (about a case). J Maroc Urol. 2008;9:34-36. 